THE COMPUTATION OF ENERGY LEVELS AND OSCILLATOR STRENGTHS

OF $\mathrm{Fe} X$ to $\mathrm{Fe} X I I I$ INVOLVING THE ADJUSTMENT

OF PARAMETERS TO ACHIEVE CONSISTENCY WITH OBSERVATIONS

\author{
B.C. Fawcett and G.E. Bromage
}

Appleton Laboratory, Astrophysics Research Division,

Science Research Council, Culham Laboratory

Abingdon, Oxon., United Kingdom

\title{
R.D. Cowan
}

The University of California, Los Alamos Scientific Laboratory, New Mexico 87545, USA.

Energy levels and oscillator strengths are calculated for the $3 s^{2} 3 p^{n}-3 s^{2} 3 p^{n-1} 3 d$ transition arrays of Fe X, Fe XI, Fe XII and Fe XIII. The Slater radial integrals for these computations are adjusted so as to become consistent with established energy levels. Pertinent configuration interactions are included and the interaction integrals are reduced by up to $35 \%$ from values calculated ab initio, to achieve consistency.

Initial theroretical calculations are made using the ab initio Hartree-X, Slater-Condon programme of Cowan $(1967,1968)$, the second of which now includes configuration interaction. The first programme computes values for the average energy of the configuration $E_{a v}$, the electrostatic direct integrals $F^{k}$ and exchange integrals $G^{k}$, the spin-orbit parameters $\zeta$ and the configuration interaction integrals $\mathrm{R}^{\mathrm{k}}$. From these the second programme computes energy levels, wavelengths and oscillator strengths for the transition arrays concerned.

Wybourne (1965) describes the well established technique of treating the radial integrals $F^{k}, G^{k}$ and $\zeta$ as empirically-adjustable parameters. The present analysis extends this method to include parametrisation of the $\mathrm{R}^{\mathrm{k}}$ configuration interaction integrals. It becomes necessary in feasible calculations to limit the number of configurations involved in the calculations because of the large size of the matrices. Separate ab initio calculations indicated which configurations mix strongly with the configuration of interest and on this basis $3 s^{2} 3 p^{n-1} 3 d, 3 s 3 p^{n+1}$, $3 s 3 p^{n-1} 3 d^{2}$ and $3 p^{n+1} 3 d$ were included. To avoid arbitrary fits the number of scaling factors are kept to a maximum of five. 
Unscaled Slater parameters for these configurations in $\mathrm{Fe} X$ to $F$ X XII give energy level values not at all consistent with observed values. In $\mathrm{Fe} X$, for instance, they can differ by up to $27,000 \mathrm{~cm}^{-1}$. whereas after scaling this error is reduced to a few hundred wavenumbers in most cases as it shown in the detailed analysis of $\mathrm{Fe} X$ and $\mathrm{Fe} X I$ by Bromage et a1. (1977). Because the ratio of the electrostatic to spin-orbit integrals is reduced relative to ab initio results, the $3 s^{2} 3 p^{n-1} 3 d$ configurations are further removed from LS towards $J j$ or JK coupling and the mixing of terms is altered. Adjustment of the $R^{k}$ factors affects the mixing of terms from different configurations. Such changes in mixing can have large effects on computed oscillator strengths; this is particularly true for intercombination or forbidden lines. The eigenvectors derived from these computations can be applied to calculations of collision strengths to make allowance for the altered mixing of the target wavefunctions

The introduction of scale factors may be merely an artifice that results from the omission of higher configurations or from other approximations inherent in the calculations. It nevertheless appears to provide better agreement between theroretical and observational data for these configurations than for other ab initio calculations feasible at the present time.

\section{$\underline{\text { References }}$}

Bromage, G.E., Cowan, R.D., Fawcett, B.C. 1977 Physics Scripta (In the press).

Cowan, R.D. 1967. Phys.Rev., 163, 54-61.

Cowan, R.D. 1968 J.0pt.Soc.Am. 58, 808-818 ibid 924-933.

Wybourne, B.G. 1965. Spectroscopic properties of rare earths, pp 39,69 (John Wiley, N.Y.). 